\title{
MYCOLOGICAL STUDY OF TINEA CAPITIS IN A TERTIARY CARE HOSPITAL
}

\author{
Anjana Gopi ${ }^{1}$, Neetha S. Murthy2, Vinoba S3, Faiza Samreen 4 , Swati ${ }^{5}$ \\ 1 Professor, Department of Microbiology, KIMS, Bangalore. \\ ${ }_{2} 3^{\text {rd }}$ Junior Resident, Department of Microbiology, KIMS, Bangalore. \\ ${ }^{3} 3^{\text {rd }}$ Junior Resident, Department of Microbiology, KIMS, Bangalore. \\ ${ }_{4}^{4} 2^{\text {nd }}$ Junior Resident, Department of Microbiology, KIMS, Bangalore. \\ $52^{\text {nd }}$ Junior Resident, Department of Microbiology, KIMS, Bangalore.
}

\begin{abstract}
BACKGROUND AND OBJECTIVE

Tinea capitis is a superficial fungal infection of the hair follicle of scalp. The causative dermatophytes vary with social status and geographical distribution. The incidence is high in developing countries due to factors like poverty compounded by overcrowding, improper hygiene and illiteracy. Tinea capitis is the most common dermatophytes infection of childhood, especially in school going children. It is self-limiting disease of childhood and seldom extends beyond puberty. India being a tropical developing country with high temperature and humidity favours superficial cutaneous mycotic infections.

The objective of this study was to isolate and identify the dermatophytes causing tinea capitis.
\end{abstract}

\section{METHOD}

A prospective study was conducted from July 2013 to August 2015. The study included 90 clinically suspected cases of tinea capitis. Among the 90 clinically suspected cases, 51 cases showed mycological evidence of fungal infection. The confirmed positive cases were then speciated based on their cultural morphology and microscopy.

\section{RESULTS}

Among the 51 culture positive cases - 20(39.21\%) Trichophyton rubrum, 14(27.45\%) Trichophyton mentagrophytes, 06(11.76\%) Trichophyton tonsurans, 05(9.8\%) Microsporum gypseum, 03(5.8\%) Microsporum canis, 02(3.92\%) Microsporum audouinii and $01(1.96 \%)$ Trichophyton violaceum species were obtained.

\section{CONCLUSION}

Trichophyton species were found to be the major causative agents for tinea capitis

\section{KEYWORDS}

Tinea Capitis, Superficial Fungal Infection, Dermatophytes.

HOW TO CITE THIS ARTICLE: Anjana Gopi, Neetha S. Murthy, Vinoba S, Faiza Samreen, Swati. "Mycological Study of Tinea Capitis in a Tertiary Care Hospital." Journal of Evolution of Medical and Dental Sciences 2015; Vol. 4, Issue 98, December 07; Page: 16295-16298, DOI: 10.14260/jemds/2015/2404

\section{INTRODUCTION}

Tinea capitis is a fungal infection of the scalp hair follicles caused by different species of dermatophytes belonging to the genera Microsporum and Trichophyton. There are over 40 species of dermatophytic fungi, of which only about 12 species are known to cause human infection. Tinea capitis is caused by approximately 6 different dermatophyte species. (1)

This superficial cutaneous mycosis can have varied clinical presentation such as inflammatory, non-inflammatory form and carrier state.(1) The non-inflammatory form can present as gray-patch scaling, seborrheic dermatitis-like scale, hair thinning without significant scaling or as distinct patches of black-dot alopecia. Inflammatory tinea capitis can present as localized pustules that are similar to impetigo and multiple widespread abscesses that mimic dissecting cellulitis. Carrier state may present as a minimal or asymptomatic infection. Tinea capitis can be transmitted via infected persons, fallen infected hair, and select animal vectors. Spread of Tinea capitis by fomites (Contaminated barbershop instruments, hairbrush and shared hats) is common.(2)

Financial or Other, Competing Interest: None.

Submission 18-11-2015, Peer Review 19-11-2015,

Acceptance 28-11-2015, Published 04-12-2015.

Corresponding Author:

Dr. Neetha S. Murthy,

L-103, $9^{\text {th }}$ B main, Sector XI, HAL-III Stage,

Jeevanbimanagar-560075,

Bangalore.

E-mail: murthyneetha4@gmail.com

DOI:10.14260/jemds/2015/2404
Those species of dermatophyte fungi most likely to cause tinea capitis vary from country to country and from region to region. Additionally, the species prevalent in a given location can change with time. In most parts of Europe, Microsporum canis has become the dominant organism in tinea capitis. However, a rise in the prevalence of Trichophyton tonsurans has been recorded in urban areas in the United Kingdom and North America. Changes in immigration patterns and increase in international travel have likely spread T. tonsurans to new areas. $\left.{ }^{3}\right)$ The exact incidence of Tinea capitis in India is unknown. It varies from $0.5 \%$ to $10 \%$.(4)

Therefore, in view of the changing trends in the causative agents, this study was undertaken to identify the predominant causative agents of Tinea capitis, the age group affected and if there is any gender preference. The study also tries to correlate various host factors with clinical manifestations.

\section{MATERIAL AND METHODS}

This study was undertaken in a Tertiary Hospital situated in the South of Bangalore between July 2013 and August 2015. All clinically suspected cases of Tinea capitis, which presented to our Dermatology Outpatient Department over a period of two years were included in the study. A detailed clinical history including age, sex, duration and type of lesion, history of close contact with affected individuals and pets was taken.

\section{Inclusion Criteria}

All new clinically suspected cases of Tinea capitis presenting with symptoms of scaling, crusting of the scalp and alopecia were included in the study. 


\section{Exclusion Criteria}

Cases of Tinea capitis on treatment.

\section{Procedure for Collection of Material}

The suspected area was cleaned with $70 \%$ alcohol and allowed to evaporate before collecting the specimen. Then the scrapings were taken from the edge of lesion with the blunt end of a sterile surgical blade (Number-15) held at an angle of $90^{\circ}$. A few affected hair strands along with their root were epilated with the help of forceps and collected in autoclaved paper, which can be stored for long periods without appreciable loss to viability of the fungal agents.

\section{Procedure for Mycological Examination}

A portion of the scraped material was placed on a clean glass slide and a drop of $10 \%$ potassium hydroxide solution was placed over it. Hair strands were placed under $20 \%$ potassium hydroxide solution. A cover slip was put over the material/hair strands. The slide was heated gently and pressed against the material, so that material was flattened and spread uniformly. The excess fluid was wiped using a filter paper. The slide was kept for 15 to 20 minutes depending on the thickness of scales. Warming on low flame was done to hasten the digestion of keratin in cases with thick scales. The prepared slide was examined under the microscope for the presence of hyphae and arthrospores.

Next, the material was inoculated into two Sabouraud's Dextrose Agar slope (SDA) one with chloramphenicol $(50 \mathrm{mg} / \mathrm{ml})+$ cycloheximide $(500 \mathrm{mg} / \mathrm{ml})$ and the other with only chloramphenicol $(50 \mathrm{mg} / \mathrm{ml})$. The inoculation was done with a sterile chromium wire spud. The tubes were labeled with patient's unique identification number and date, following which the inoculated tubes were incubated at room temperature. The tubes were examined every day for evidence of any growth. They were discarded as negative if there was no growth after 4 weeks.

\section{In case of a growth, the following parameters were looked into:}

- Rate of growth, General topography and characteristics of colony (i.e., yeast like powdery granular velvety or cottony pigmentation on the surface and on reverse.)

Lactophenol Cotton Blue (LPCB) mount was prepared from the growth to study the exact morphological characteristics of the fungi isolated. Slide culture was put up for isolates where the morphology was not clearly distinguishable on LPCB mounts to aid the process of identification.

\section{RESULTS}

Out of the 90 clinically suspected cases, 51 (56.66\%) showed mycological evidence of tinea capitis. The age-wise split-up of the cases showed that the highest incidence was accounted between the ages 1-10 years (56.86\%). (Table-1).

Out of the 51 cases, $28(54.90 \%)$ were males and $23(45.09 \%)$ were females. (Figure-1).

Grey patch (54.90\%) was the commonest clinical type, followed by seborrhoeic dermatitis like presentation. (25.49\%) (Table-2/Figure 2-4).

Out of the 90 clinically suspected cases, $\mathrm{KOH}$ microscopy was positive in 38(42.22\%) individuals and 51(56.66\%) were positive by culture (Table-3). Significant difference in the positivity rates of $\mathrm{KOH}$ and culture was observed.

Trichophyton rubrum was the commonest species $(39.2 \%)$ isolated followed by T. mentagrophytes $(27.4 \%)$ and T. tonsurans (11.7\%) (Table 4). Maximum number of isolates was from grey patch.

\section{DISCUSSION}

The present study showed dermatophyte isolation rate of $56.66 \%$ among the suspected clinical cases. Isolation rates ranging from $24 \%-93 \%$ have been reported in earlier studies from different geographical areas. $(5,6,7)$ Our study showed $\mathrm{KOH}$ positivity rate of $42.2 \%$ among the clinically suspected cases, which was less compared with earlier studies by Jha BN et al. and Woldeamanuel Y et al., where in the rates were $62.3 \%$ and $62 \%$ respectively; however, the $\mathrm{KOH}$ positivity rate was much higher when compared to $16.3 \%$ by Hogewoning AA et al. $(8,9,10)$ According to our study, the predominant age group affected was found to be 1-10 (56.86\%). Similar findings have been noted in studies by A. G. Sajjan et al., Bose S. et al., Wani M.M. et al. and Pai et al.(11,12,6,4) The decreased incidence after puberty is believed to be due to the sebum containing fungistatic free fatty acids.(13)

The most common clinical presentation was the grey patch followed by seborrhoeic dermatitis like presentation. Similar findings have been noted by Jha B.N., et al. and A. G. Sajjan et al. $(8,11)$ However, Bose S. et al. has reported seborrheic variant as the predominant type followed by black dot and kerion.(12)

Statistically, there was no significant difference between the number of females affected as against the number of males affected in this study. Studies done in Trinidad and Tobago showed significantly more males affected (55 males of 70 cases) with tinea capitis infection.(14) A New York based study also showed a dominance of males in the paediatric patients (With 45 males of 70 cases), the majority of whom were of African-American extract.(15) The factors contributing to the cause of infection in males and females may differ. Factors which may make females vulnerable to infection are more elaborate hairstyles involving increased traction like braids, which may lead to less frequent shampooing and the traction increases the susceptibility of the hair follicle to infection.(15)

The role of the woman as the primary caretaker of children in the household may also increase the susceptibility in female adults.(15) For males on the other hand, barbers must be considered as a possible reservoir for the disease.(15) The adoption by men of hairstyles, which increase traction such as braids may lead to less frequent shampooing, therefore make them more vulnerable to tinea capitis. Two attributes favoring the increased incidence of tinea capitis in males are greater physical activity leading to increased sweating and the presence of short hair in males, which favour the spores to reach the scalp easily. $(16,13)$

During our study we had 3 cases of M. canis (Figure-5) associated tinea capitis. Two cases were from the same household and they owned a Persian cat. One another case which was positive for M. canis also owned a pet dog. M. canis is a zoophilic fungus, therefore pets can be traced out to be the probable focus of infection. M. canis can be transmitted from human to human, but it may lose its virulence after about four human to human transmissions; thus, an animal host is necessary to regain the virulence.(17) Zoophilic fungi cause a brisk inflammatory response in nearly all infected people and are less likely to cause an asymptomatic carrier state.(18)

Isolation of different species of dermatophytes also depends on the ecological niche. Trichophyton rubrum was the commonest species isolated in our study and also in studies from various parts of India done by Kannan et al, A. G. Sajjan et al., Jain N. et al.(19,11,16) This result does not match with earlier studies done in Nepal and Ethiopia, which show T. violaceum was the most frequent species isolated, $(8,9)$ therefore showing the possible geographical variation of dermatophytes. 


\section{CONCLUSION}

In conclusion, the present study showed that culture was more sensitive as compared to $\mathrm{KOH}$ mount examination and the commonest species isolated was T. Rubrum. The results of this study emphasize that tinea capitis is a superficial fungal infection seen predominantly in children. The tropical warm and humid climate, pets and poor sanitary conditions augment the spread of this infection.

\section{ACKNOWLEDGEMENTS}

We extend our gratitude to Dr. Sharath Kumar B.C, Professor and Head of the department of dermatology, KIMS RC\&H for referring the patients for the study and for his valuable suggestions.

\section{REFERENCES}

1. Elewski BE. Tinea capitis - A current perspective. J Am Acad Dermatol 2000;42:1-19.

2. Mackenzie DW. 'Hairbrush diagnosis' in detection and eradication of non-fluorescent scalp ringworm. Br Med J 1963;11:363-5.

3. East-Innis A, Rainford L, Dunwell P, Barrett-Robinson D, Nicholson AM. The changing pattern of Tinea capitis in Jamaica. West Indian Med J 2006 Mar;55(2):85-88.

4. Pai VV, Hanumanthayya K, Tophakhane RS, Nandihal NW, Kikkeri NN. Clinical study of Tinea capitis in Northern Karnataka: A three-year experience at a single institute. Indian Dermatol Online J 2013;4:22-6.

5. Grover C, Arora P, Manchanda V. Tinea capitis in the pediatric population: A study from North India. Indian J Dermatol Venereol Leprol 2010;76:527-32.

6. Wani MM, Kamili QA, Chisti M, Wani MM \& Masood Q. Trends of tinea capitis in population attending Dermatology Department of a Tertiary Healthcare Facility in Kashmir. JK-Practitioner 2006;13:131-133.

7. Azab MM, Mahmoud NF, Abd Allah S, Alaa El Din, Hosny MS, Shehata AS and Mohamed RW. Dermatophytes isolated from clinical samples of children suffering from tinea capitis in Ismailia, Egypt. Aust J basic appl sci 2012;6:38-42.

8. Jha BN, Garg VK, Agrawal S, Khanal B, Agarwalla A. Tinea capitis in Eastern Nepal. Int J Dermatol

2006;45:100-102.

9. Woldeamanuel Y, Leekassa R, Chryssanthou E, Menghistu Y and Petrini B. Prevalence of tinea capitis in Ethiopian school children. Mycoses 2005;48:137-141.
10. Hogewoning AA, Adegnika AA, Bouwes Bavinck JN, Yazdanbakhsh M, Kremsner PG, Van Der Raaij-Helmer EMH, Staats CCG, Willemze R and Lavrijsen APM. Prevalence and causative fungal species of tinea capitis among school children in Gabon. Mycoses 2011;54(5):354-359.

11. Sajjan AG, Mangalgi SS. Clinicomycological profile of tinea capitis in children residing in orphanages. Int J Biol Med Res 2012;3(4):2405-2407.

12. Bose S, Kulkarni SG, Akhter I. The incidence of tinea capitis in a Tertiary Care Rural Hospital - a study. Journal of Clinical and Diagnostic Research 2011;5:307-311.

13. Kanwar AJ, De D. Superficial fungal infections. In: Valia RG, Valia AR, editors. IADVL textbook of Dermatology. 3 rd ed. Mumbai: Bhalani publishing house 2008. p. 252-97.

14. Moore MK, Suite M. Tinea capitis in Trinidad. J Trop Med Hyg 1993;96:346-8.

15. Silverberg NB, Weinberg JM, De Leo VA. Tinea capitis: focus on African American women. J Am Acad Dermatol 2002;46(Suppl 2):120-4.

16. Jain N, Sharma M, Saxena VN. Clinico-mycological profile of dermatophytosis in Jaipur, Rajasthan. Indian J Dermatol Venereol Leprol 2008;74:274-5.

17. Aly R. Ecology, epidemiology and diagnosis of tinea capitis. Pediatr Infect Dis J 1999;18(2):180-185.

18. Frieden IJ. Tinea capitis: asymptomatic carriage of infection. Pediatr Infect. Dis J 1999;18:186-190.

19. Kannan P, Janaki C, Selvi GS. Indian J of Med Microbiol 2006;24:212-215.

\begin{tabular}{|c|c|c|c|c|c|}
\hline $\begin{array}{c}1-10 \\
\text { years }\end{array}$ & $\begin{array}{c}11-20 \\
\text { years }\end{array}$ & $\begin{array}{c}\mathbf{2 1 - 3 0} \\
\text { years }\end{array}$ & $\begin{array}{c}\mathbf{3 1 - 4 0} \\
\text { years }\end{array}$ & $\begin{array}{c}\mathbf{4 0 - 5 0} \\
\text { years }\end{array}$ & $\begin{array}{c}>\mathbf{5 0} \\
\text { years }\end{array}$ \\
\hline 29 & 07 & 05 & 06 & 02 & 02 \\
\hline \multicolumn{6}{|c|}{ Table 1: Age-wise distribution } \\
\hline
\end{tabular}

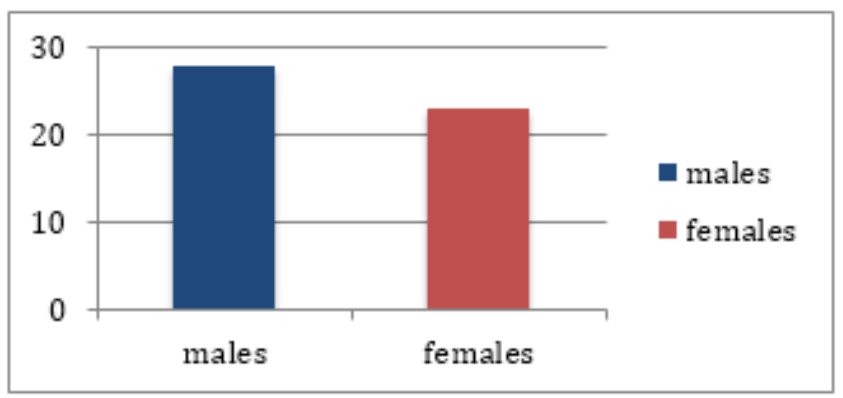

Fig. 1: Gender-wise distribution

\begin{tabular}{|c|c|c|c|c|c|c|}
\hline Isolates & Grey patch & Black dot & Favus & Kerion & Seborrhoeic dermatitis & Total \\
\hline T. rubrum & 12 & - & - & 01 & 07 & $20(39.2 \%)$ \\
\hline T. mentagrophytes & 11 & - & - & 02 & 01 & $14(27.4 \%)$ \\
\hline T. tonsurans & - & 04 & - & - & 02 & $06(11.7 \%)$ \\
\hline M. gypseum & 02 & & 01 & & 02 & $05(9.8 \%)$ \\
\hline M. canis & - & - & - & 02 & 01 & $03(5.8 \%)$ \\
\hline M.audouinii & 02 & - & - & - & - & $02(3.9 \%)$ \\
\hline T.violaceum & 01 & - & - & - & - & $01(1.9 \%)$ \\
\hline Total & $\mathbf{2 8}$ & $\mathbf{0 4}$ & $\mathbf{0 1}$ & $\mathbf{0 5}$ & $\mathbf{1 3}$ & $\mathbf{5 1}$ \\
\hline \multicolumn{7}{|l}{} \\
\hline
\end{tabular}




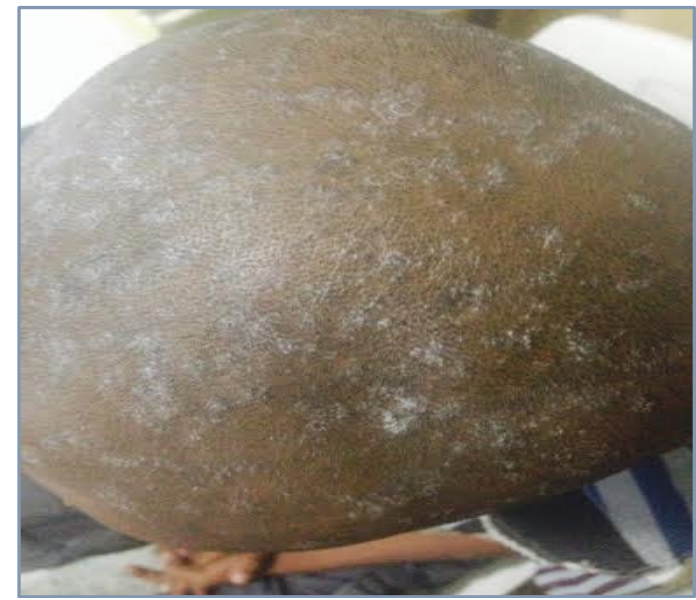

Fig. 2: Grey patch type of tinea capitis

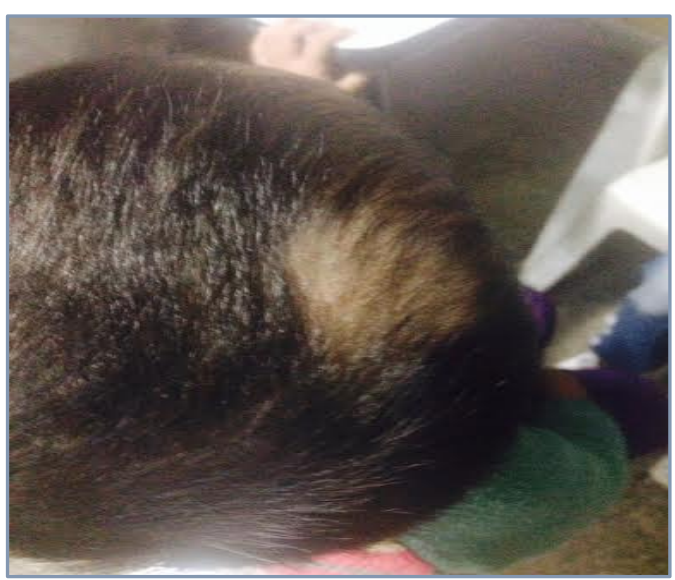

Fig. 3: Blackdot type of tinea capitis

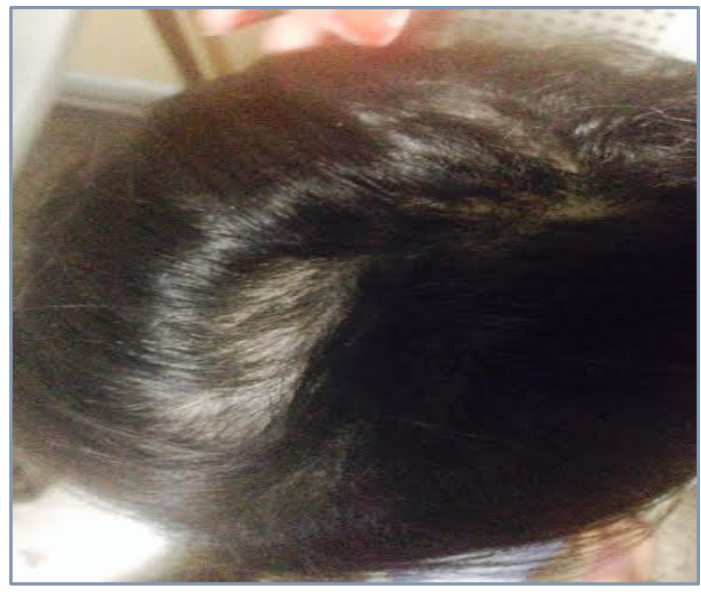

Fig. 4: Seborrhoeic dermatitis type of tinea capitis

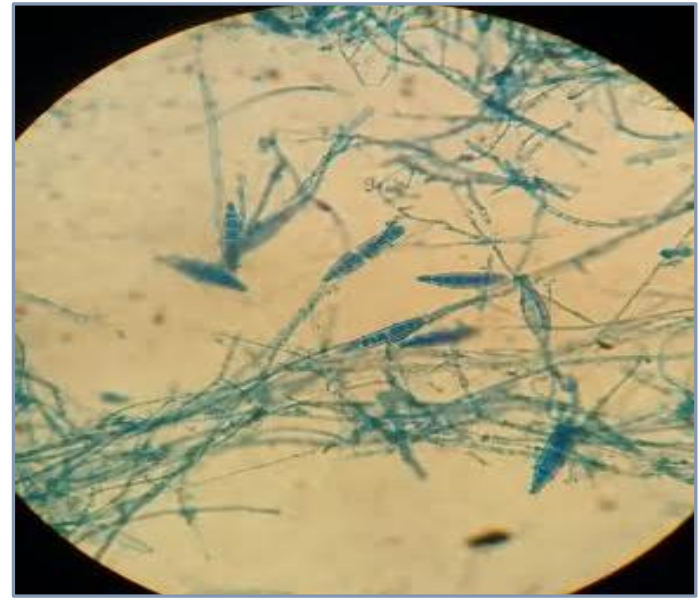

Fig. 5: Microscopic picture of the LPCB mount of M.canis isolated

\begin{tabular}{|c|c|c|c|c|c|}
\hline $\begin{array}{c}\text { Total } \\
\text { suspected } \\
\text { cases }\end{array}$ & $\begin{array}{c}\text { Confirmed } \\
\text { tinea capitis } \\
\text { cases }\end{array}$ & $\begin{array}{c}\text { KOH and } \\
\text { culture } \\
\text { positive }\end{array}$ & $\begin{array}{c}\text { KOH negative } \\
\text { culture positive }\end{array}$ & $\begin{array}{c}\text { KOH and } \\
\text { culture } \\
\text { negative }\end{array}$ & $\begin{array}{c}\text { KOH positive } \\
\text { culture } \\
\text { negative }\end{array}$ \\
\hline 90 & 51 & 31 & 20 & 32 & 07 \\
\hline \multicolumn{5}{|c|}{ Table 3: Comparison of KOH mount \& culture results } \\
\hline
\end{tabular}

